\title{
Pesquisa da função vestibular em crianças com queixa de dificuldades escolares
}

Vestibular function in children underperforming at school

\author{
Eloisa Sartori Franco ${ }^{1}$, Ivone Panboca ${ }^{2}$
}

Palavras-chave: aprendizagem, doenças do labirinto, vertigem.

Keywords: learning, labyrinthine disease, vertigo.

\section{Resumo / Summary}

$\mathrm{O}$

aprendizado é um processo complexo, dinâmico, estruturado a partir de um ato motor e perceptivo, que, elaborado corticalmente, dá origem à cognição. O equilíbrio é função neurológica importante para a manutenção de posturas adequadas, imprescindíveis no ato de aprender, indicativo de maturidade neurológica. Objetivo: Estudar a função vestibular em crianças com dificuldades escolares. Estudo de Caso: Estudo clínico com coorte transversal. Material e Método: Foram estudadas 88 crianças entre 7 e 12 anos, que freqüentavam escolas públicas da cidade de Piracicaba durante os anos de 2004 e 2006. Os procedimentos utilizados foram: a anamnese; exame otorrinolaringológico; exame audiológico e avaliação vestibular. Resultados: Das crianças avaliadas, 51,0\% não relataram dificuldades escolares e 49,0\% referiram ter dificuldades escolares. Encontramos $73,3 \%$ de exame vestibular normal nas crianças sem dificuldades escolares e 32,6\% de normalidade nas crianças com dificuldades escolares. Encontramos alterações vestibulares de origem periférica irritativa tanto unilateral como bilateral, perfazendo um total de $67,4 \%$ para as crianças com dificuldades escolares e um total de $26,7 \%$ para crianças sem dificuldades escolares. Conclusão: Todas as alterações vestibulares encontradas foram de origem periférica irritativa. Os dados revelaram uma relação estatisticamente significante nas crianças com dificuldades escolares.

\begin{abstract}
L ing a complex, dynamic process, structurec from motor and perception skills which, when cortically processed, give birth to cognition. Balance is a fundamental neurological function that helps us maintain proper postures, an essential factor in learning and a sign or neurologic maturity. Aim: this paper aims to study vestibular function in children underperforming at school. Study design: this is a cross-sectional study. Materials and method: eightyeight children with ages ranging between 7 and 12 years attending the public schools of Piracicaba from 2004 to 2006 were enrolled. All children were interviewed, submitted to ENT examination, hearing tests, and vestibular examination. Results: fifty-one percent of the participants had no reported difficulties at school, whereas $49.0 \%$ were underperforming at school. Under vestibular examination, $73.3 \%$ of the children performing well at school had normal findings, whereas $32.6 \%$ of the underperforming children had normal test results. Unilateral and bilateral irritative peripheral vestibular alterations were found in $67.4 \%$ of the underperformers and in $26.7 \%$ of the children not experiencing difficulties at school. Conclusion: all vestibular alterations found had an irritative peripheral origin. There was a statistically significant association between vestibular alteration and poor performance at school.
\end{abstract}

\footnotetext{
${ }^{1}$ Mestre em Fonoaudiologia pela PUC - SP. Docente do Curso de Fonoaudiologia da Universidade Metodista de Piracicaba - UNIMEP. Supervisora de Estágios em Audiologia - UNIMEP.

${ }^{2}$ Doutora em Ciências pelo Instituto de Estudo de Linguagem da UNICAMP. Docente da Faculdade de Fonoaudiologia da PUC - Campinas. Orientadora credenciada externa do Programa "Saúde da Criança e do Adolescente", Depto de Pediatria, FCM/UNICAMP.

Instituição ao qual o trabalho está vinculado: Programa de Pós-Graduação em Saúde da Criança e do Adolescente - Departamento de Pediatria - FCM/UNICAMP. Instituição em que foi realizado o trabalho: Faculdade de Ciências da Saúde - FACIS - Curso de Fonoaudiologia - UNIMEP.

Endereço para correspondência: Eloisa Sartori Franco - R. Aldeia Campista 103 - Cond. San Conrado Sousas Campinas SP 13104-051. Este artigo foi submetido no SGP (Sistema de Gestão de Publicações) da RBORL em 25 de junho de 2007. cod.4627

Artigo aceito em 21 de outubro de 2007.
} 


\section{INTRODUÇÃO}

O aprendizado é um processo complexo, dinâmico, estruturado a partir de um ato motor e perceptivo que, elaborado corticalmente, dá origem à cognição. Os distúrbios de áreas específicas do sistema nervoso central, relacionadas com a noção do esquema corporal, do espaço e do tempo, constituem as bases neuropatológicas das alterações perceptomotoras ou dispatognósicas, das quais poderiam resultar os quadros de dificuldade de aprendizagem ${ }^{1}$.

Um grupo de 103 crianças com e sem dificuldades de aprendizagem escolar foi avaliado sendo que, 60 crianças $(58,2 \%)$ não relataram dificuldades e 43 crianças $(41,7 \%)$ relataram dificuldades escolares ${ }^{2}$.

Fatores genéticos, afecções sensoriais periféricas, afecções neurogênicas, afecções médicas gerais, desvantagem sócio-cultural e transtornos pedagógicos podem interferir negativamente no processo de aprendizagem ${ }^{3}$.

Temos os transtornos das integrações funcionais básicas, do esquema corporal, da orientação espacial, do ritmo, da coordenação motora fina, da lateralidade, da função análise e síntese, da integração das partes em um todo, da simbolização, da linguagem em seus diferentes aspectos, dos impulsos, da atenção e da memória, perturbações neurogênicas essas, que podem produzir alterações no aprendizado ${ }^{4}$.

Uma função neurológica importante para a manutenção de posturas adequadas e imprescindíveis para o ato de aprender é o equilíbrio estático. Temos o equilíbrio dinâmico que é, também, uma função evolutiva importante que proporciona indicativos de maturidade neurológica. Pesquisas relatam que crianças com imaturidade desta função têm mais probabilidade de apresentar dificuldades de aprendizado5.

O sistema vestibular, os proprioceptores e o cerebelo são responsáveis por funções como tônus muscular, postura, equilỉbrio, coordenação oculomotora e orientação espacial. Este sistema parece estar grandemente envolvido no processo de aprendizagem escolar ${ }^{6}$.

Narciso et al. ${ }^{7}$ pesquisaram alterações vestibulares em crianças, observando que $47 \%$ delas apresentavam queixas no desempenho escolar. Constataram que os distúrbios vestibulares podem estar associados às dificuldades escolares e motoras.

Segundo Campos et al. ${ }^{8}$ a disfunção vestibular infantil pode afetar consideravelmente a habilidade de comunicação, o estado psicológico e o desempenho escolar, sendo importante lembrar que o baixo rendimento escolar também pode ser um indício valioso de possível labirintopatia.

De acordo com Caovilla et al. ${ }^{9}$ o reconhecimento precoce das vestibulopatias infantis e o seu tratamento etiológico são essenciais na prevenção das complicações que freqüentemente decorrem no desenvolvimento motor e na aquisição de linguagem. Deve-se fazer o exame vestibular em toda a criança que apresentar uma forte suspeita de disfunção vestibular, muito embora não seja fácil obter da criança ou de seus pais uma descrição precisa dos sintomas.

O exame funcional do sistema vestibular pode ser realizado por meio da eletronistagmografia ou vectonistagmografia A vectonistagmografia digital é um dos métodos mais empregados no nosso meio para avaliar a função vestibular, conferindo maior sensibilidade diagnóstica por permitir a medida dos parâmetros da função vestíbulooculomotora à comparação entre estímulos e respostas, além de identificar a direção dos fenômenos ${ }^{10}$.

Ganança et al. ${ }^{11}$ estudaram 30 crianças com queixas de dificuldades escolares, encontrando alterações à nistagmografia computadorizada em $20,0 \%$ das crianças avaliadas.

Os testes que compõe a vectonistagmografia digital segundo Caovilla et al. ${ }^{12}$ são os testes oculomotores (analisando os movimentos oculares, o nistagmo espontâneo e semi-espontâneo, os movimentos sacádicos, o rastreio pendular e o nistagmo optocinético) e os testes vestibulares (estimulação rotatória e estimulação térmica).

Anormalidades no controle voluntário da sacade têm sido observadas em muitas desordens do desenvolvimento tais como: a dislexia; dificuldades de aprendizagem; hiperatividade e déficit de atenção ${ }^{13}$.

O movimento ocular necessário para uma leitura exige movimentos alternados de sácade e períodos de fixação. Inicia-se com uma sácade que percorre de 8 a 10 palavras mescladas com períodos de fixação ocular e finaliza-se com uma longa sácade para reiniciar uma nova linha ${ }^{14}$.

A via sacádica envolve várias regiões do córtex cerebral, cerebelo e tronco cerebral. Os parâmetros latência, velocidade e precisão dos movimentos sacádicos avaliam a eficiência do controle do sistema nervoso central (SNC) sobre os movimentos rápidos dos olhos. Poucas desordens que alteram o SNC deixam de ser detectadas quando latência, velocidade e precisão dos movimentos sacádicos são medidas com rigor por meio de um computador ${ }^{10}$.

As alterações vestibulares encontradas em crianças disléxicas levaram os autores Frank e Levinson ${ }^{15}$ a emitirem a hipótese de que uma disfunção vestibular e um nistagmo espontâneo poderiam interferir nas fixações oculares seqüenciais necessárias à leitura.

O rastreio pendular é outro movimento dos olhos resultante do acompanhamento de um alvo móvel e avalia a integridade do sistema oculomotor no controle dos movimentos oculares lentos, vulneráveis a disfunções do SNC e do sistema vestibular.

Acompanhar a professora, em seu campo visual, na sala de aula, fazer cópias, transcrever as lições escritas na 
lousa, ler as lições do livro, escrever e concentrar-se são atividades que exigem integridade das funções oculomotoras e das interligações vestibulares.

O nistagmo optocinético é um fenômeno ocular rítmico, involuntário, inconsciente e automático. Pode ser reproduzido ao acompanhar pontos que se movem em uma direção e depois na direção oposta. Representa uma resposta exteroceptiva que compensa os movimentos do meio ambiente por impulsos psico-ópticos. O nistagmo optocinético pode estar alterado nas síndromes do SNC e nas disfunções vestibulares ${ }^{10}$.

Horak et al. ${ }^{16}$ estudaram a função vestibular em crianças com e sem dificuldades de leitura e escrita, encontrando alteração no reflexo vestíbulo ocular em 20\% das crianças com dificuldades escolares e constatando que $7 \%$ das crianças sem queixas escolares apresentavam alterações vestibulares.

A estimulação rotatória realizada na prova rotatória pendular decrescente permite avaliar o estado de compensação labiríntica analisando sua preponderância direcional ${ }^{17}$.

Ayres $^{18}$ estudou os movimentos nistagmicos na estimulação pós-rotatória em crianças com dificuldades de aprendizagem e verificou nistagmos pós-rotatório diminuído em $50 \%$ das crianças com dificuldades de aprendizagem e nistagmos pós-rotatório exacerbado em 13\% delas.

A estimulação térmica, realizada por meio da prova calórica, é a prova de maior sensibilidade na bateria de testes vestibulares convencionais possibilitando o encontro de alterações vestibulares em pacientes sem queixas específicas e sem anormalidades nas outras etapas da avaliação vestibular.

Ganança et al. ${ }^{11}$ estudaram 30 crianças com queixas de dificuldades escolares, encontrando alterações à nistagmografica computadorizada em $20,0 \%$ das crianças avaliadas, sendo todas elas de topodiagnóstico periférico. Segundo os autores, a prova calórica foi o teste vestibular que apresentou o maior número de alterações.

Ganança ${ }^{19}$ realizou um estudo com 64 crianças com distúrbio de linguagem e sem queixas de tonturas encontrando alta incidência de alterações vestibulares de topodiagnóstico periférico.

Dados relevantes foram observados nos estudos de Franco e Caetanelli ${ }^{20}$ que realizaram avaliação vestibular em crianças em idade escolar por meio da vectonistagmografia computadorizada e concluíram que 20,7\% delas, apresentaram alterações vestibulares de caráter periférico irritativo, tanto unilateral quanto bilateral.

Os estímulos sensoriais são requisitos fundamentais para o desenvolvimento e aprendizagem da criança. Estimulada desde cedo, a criança terá boa coordenação, concentração e será criativa, tendo grandes chances de tornar-se um ser pensante, autoconfiante sem dificuldades na aprendizagem.

A presente pesquisa teve como objetivo estudar a função vestibular das crianças em idade escolar com dificuldades escolares tornando possível uma melhor compreensão das desordens vestibulares desencadeantes e propiciando, como conseqüência, estudos futuros que redundem em programas de intervenção precoce.

\section{MATERIAL E MÉTODO}

\section{Tipo de estudo}

O presente estudo, de caráter experimental, uma vez aprovado pelo Comitê de Ética (Processo 423/2003), foi realizado utilizando as dependências da Clínica-Escola de uma Universidade do interior de São Paulo, bem como seus equipamentos.

\section{Seleção dos casos}

Foram sujeitos da amostra crianças entre 7 e 12 anos, que freqüentavam escolas públicas da cidade de Piracicaba e foram convidados a comparecerem à ClínicaEscola durante os anos de 2004 e 2006.

Avaliamos separadamente 2 grupos de crianças, sendo um grupo de crianças que não referiram dificuldades escolares e um grupo de crianças que referiram dificuldades escolares. Tais referências como: não adquirir, junto aos colegas, novos conhecimentos; não acompanhar as atividades propostas em sala de aula; histórico de retenção entre outras, foram obtidas a partir de uma anamnese com as crianças juntamente com seus pais e/ou responsáveis, definindo-se a que grupo as crianças pertenceriam.

Para a realização da presente pesquisa foram convidados 100 escolares acompanhados de seus pais que, encaminhados a Clínica-Escola, foram orientados quanto à participação no estudo.

Foi realizada uma reunião elucidativa em data, local e horário acordado, quando foi distribuído a cada participante uma carta de informação e o termo de consentimento livre e esclarecido. Todos os escolares dessa pesquisa foram agendados, após autorização dos responsáveis. Todos os sujeitos envolvidos consentiram na realização desta pesquisa e a divulgação de seus resultados conforme a Resolução 196/96.

- Critério de inclusão - grupo com queixa de dificuldades escolares: foram incluídas na amostra as crianças que referiram queixas de dificuldades escolares; apresentaram limiares auditivos tonais de $500 \mathrm{~Hz}$ a $8000 \mathrm{~Hz}$, inferiores ou iguais a $15 \mathrm{dBNA}^{21,22}$; timpanometria tipo A e reflexos acústicos contralateral e ipsilateral presentes bilateralmente nas freqüências de $500 \mathrm{~Hz}$ a $4000 \mathrm{~Hz}^{23}$.

- Critério de inclusão - grupo sem queixa de dificuldades escolares: foram incluídas na amostra as crianças que não referiram queixas de dificuldades escolares; apresentaram limiares auditivos tonais de $500 \mathrm{~Hz}$ a $8000 \mathrm{~Hz}$, inferiores ou iguais a $15 \mathrm{dBNA}^{21,22}$; timpanometria tipo A e reflexos acústicos contralateral e ipsilateral presentes 
bilateralmente nas freqüências de $500 \mathrm{~Hz}$ a $4000 \mathrm{~Hz}^{23}$.

- Critério de exclusão - ambos os grupos: foram excluídas as crianças menores de 7 anos e maiores de 12 anos, que relataram sintomas ou alterações auditivas e visuais que pudessem interferir nos resultados desta pesquisa.

\section{PROCEDIMENTOS}

\section{Anamnese:}

Inicialmente foi realizada uma anamnese com todas as crianças e seus pais, visando à obtenção de dados referentes às queixas de origem vestibular dando ênfase à presença ou não de vertigem, explorando queixas associadas, principalmente quanto aos aspectos auditivos, sintomas neurovegetativos, casos neurológicos com suspeita de comprometimento da fossa posterior.

\section{Exame otorrinolaringológico:}

Com a contribuição voluntária do médico Otorrinolaringologista, ambos os grupos foram submetidos a exame otorrinolaringológico com o objetivo de afastar a hipótese de qualquer desordem de orelha, nariz e garganta que pudesse repercutir sobre o sistema auditivo e vestibular.

\section{Exame audiológico:}

A avaliação audiológica consistiu na realização da audiometria tonal limiar por via aérea e por via óssea quando necessário, pesquisa do índice percentual de reconhecimento de fala, do limiar de reconhecimento de fala e análise da imitância acústica, de acordo com os critérios de Mangabeira Albernaz et al. ${ }^{23}$. Para a realização da audiometria tonal e vocal, em cabina acusticamente tratada, foi utilizado o audiômetro da marca MADSEN MIDIMATE 622 e para a imitanciometria o analisador de orelha média MADSEN ZO-72.

Foram adotados os critérios de Glorig e Davis ${ }^{21}$ e de Mangabeira Albernaz et al. ${ }^{22}$ que consideram os padrões normais da audição em várias idades para a caracterização dos limites da audição normal.

Os resultados do estudo audiológico foram utilizados apenas para critério de inclusão.

\section{Exame vestibular:}

Para arealização do exame vestibular as crianças desta pesquisa foram instruídas ase absterem de café, chá, chocolate ou qualquer medicamento estimulante labirínti$\mathrm{co}$, durante as 72 horas que antecederem o exame.

O exame vestibular foi realizado seguindo-se as orientações de Caovilla et al. ${ }^{12}$ tanto na seqüência de realização quanto nos parâmetros de interpretação das provas vestibulares. A interpretação dos resultados do exame vestibular seguiu os parâmetros determinados por Ganança et al. ${ }^{24}$.
O Registro vectonistagmográfico foi realizado com o auxílio do vectonistagmógrafo digital computadorizado (sistema VECWIN) que capta e grava em três canais de registro, estimulador visual modelo - EVR 03 e otocalorímetro a ar modelo - NGR 05 da marca NEUROGRAFF ELETROMEDICINA LTDA.

Dessa maneira, todos foram submetidos à:

Pesquisa do nistagmo ou vertigem de posição

Seguindo os preceitos de Caovilla et al. ${ }^{12}$.

Calibração biológica dos movimentos oculares

Para a calibração dos movimentos oculares foi utilizado o calibrador biológico (estimulador visual).

Pesquisa do nistagmo espontâneoe semi-espontâneo

Para a pesquisa do nistagmo espontâneo foi utilizada a barra de luzes (estimulador visual).

Pesquisa dos movimentos sacádicos

Para essa pesquisa foi utilizada a barra de luzes (estimulador visual).

Pesquisa do rastreio pendular

Para essa pesquisa foi utilizada a barra de luzes (estimulador visual).

Pesquisa do nistagmo optocinético

Para essa pesquisa foi utilizada a barra de luzes (estimulador visual).

Pesquisa do nistagmo per-rotatório

Nesta prova, utilizamos a cadeira rotatória pendular da marca YOSHI.

Pesquisa do nistagmo pós-calórico

Para a pesquisa do nistagmo pós-calórico, utilizamos o otocalorímetro a ar.

Parâmetros de avaliação

Segundo as orientações de Caovilla et al. ${ }^{12}$, procedemos à análise, que são de interesse para a semiologia da função vestibular.

\section{Critérios para Análise:}

A interpretação dos resultados do exame vestibular seguiu os parâmetros determinados por Ganança et al. ${ }^{24}$.

\section{Método estatístico:}

A análise dos resultados pertinentes à avaliação vestibular, em função da natureza das variáveis estudadas, efetuamos usando-se os seguintes testes:

- paramétricos: Teste t de Student, controlado pelo Teste de Levene ${ }^{25}$, buscando comparar as médias entre duas variáveis estudadas;

- não paramétricos: Teste de Mann-Whitney ${ }^{26}$ verificando associações entre as variáveis.

Obedecemos aos limites de confiança de 95\%, baseados nos valores de media e desvio-pa-drão para as variáveis estudadas.

Em todos os testes fixamos em 0,05 ou $5 \%(a \leq 0,05)$ 
para o nível para rejeição da hipótese de nulidade e destacaremos os valores significantes.

Usamos o programa SPSS (Statistical Package for Social Sciences) em sua versão 13.0 para obtenção dos resultados.

\section{RESULTADOS}

Na Tabela 1 pode ser visualizada a distribuição da amostra quanto aos percentuais do desempenho escolar em função do sexo.

Tabela 1. Distribuição da amostra em relação ao sexo e desempenho escolar $(n=88)$.

\begin{tabular}{cccc}
\hline \multirow{2}{*}{$\begin{array}{c}\text { Dificuldade } \\
\text { escolar }\end{array}$} & \multicolumn{2}{c}{ sexo } & Total \\
\cline { 2 - 3 } & feminino & masculino & \\
\hline \multirow{2}{*}{ Não } & 22 & 23 & 45 \\
& $48,9 \%$ & $51,1 \%$ & $100,0 \%$ \\
Sim & 14 & 29 & 43 \\
& $32,6 \%$ & $67,4 \%$ & $100,0 \%$ \\
Total & 36 & 52 & 88 \\
& $40,9 \%$ & $59,1 \%$ & $100,0 \%$ \\
\hline
\end{tabular}

$p=0,121$

Na Tabela 2 dispomos o resultado do Teste t de Student, controlado pelo Teste de Levene para Igualdade de Variâncias, para verificarmos possíveis diferenças entre as médias das variáveis paramétricas para os parâmetros oculomotores da calibração: latência; velocidade e precisão.

Tabela 2. Distribuição da amostra em relação aos parâmetros oculomotores da calibração e desempenho escolar $(n=88)$.

\begin{tabular}{cccccc}
\hline Variável & dificuldade & $n$ & Média & $\begin{array}{c}\text { Des- } \\
\text { vio-pa- } \\
\text { drão }\end{array}$ & $\begin{array}{c}\text { Significân- } \\
\text { cia (p) }\end{array}$ \\
\hline latência_D_1 & sim & 43 & 157,00 & 81,68 & 0,531 \\
velocidade_D_1 & não & 45 & 147,31 & 90,25 & \\
precisão_D_1 & não & 43 & 160,35 & 50,32 & 0,460 \\
& sim & 43 & 151,13 & 50,42 & \\
latência_E_1 & não & 45 & 84,99 & 22,23 & 0,822 \\
& sim & 43 & 188,36 & 98,34 & 0,438 \\
velocidade_E_1 & não & 45 & 169,52 & 85,17 & \\
& sim & 43 & 143,84 & 51,52 & 0,515 \\
precisão_E_1 & não & 45 & 151,05 & 53,64 & \\
& sim & 43 & 87,18 & 17,95 & 0,796 \\
\hline
\end{tabular}

$a \leq 0,05$
A seguir, na Figura 1 representamos, em boxplot, o estudo estatístico para o parâmetro oculomotor da calibração: latência.

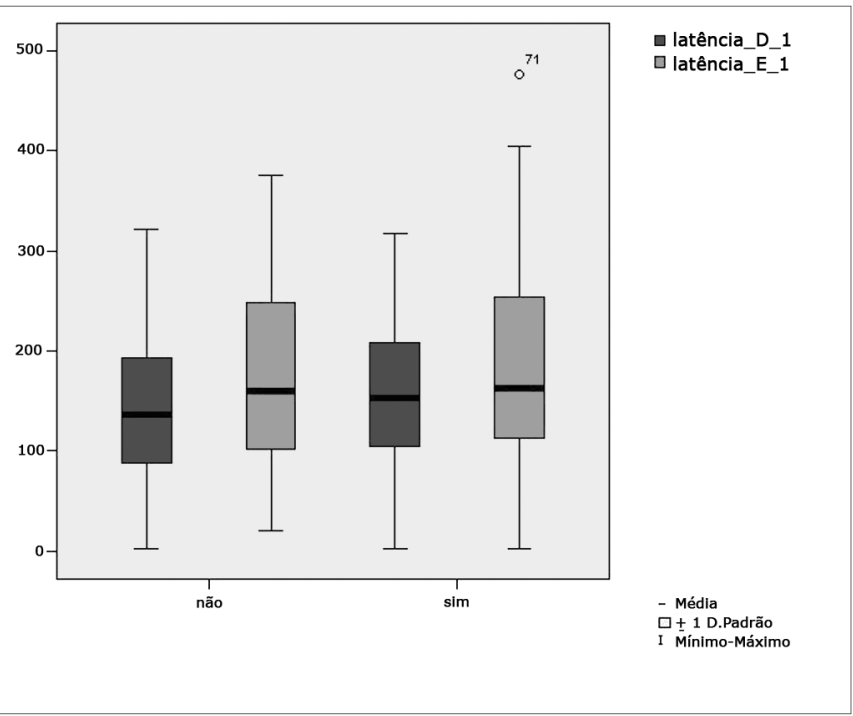

Figura 1. Boxplot para o parâmetro oculomotor de calibração (latência) direita e esquerda, por grupo. (Teste t de Student, controlado pelo Teste de Levene para Igualdade de Variâncias, entre as médias das variáveis paramétricas de interesse).

Na Figura 2 representamos, em boxplot, o estudo estatístico para o parâmetro oculomotor da calibração: velocidade.

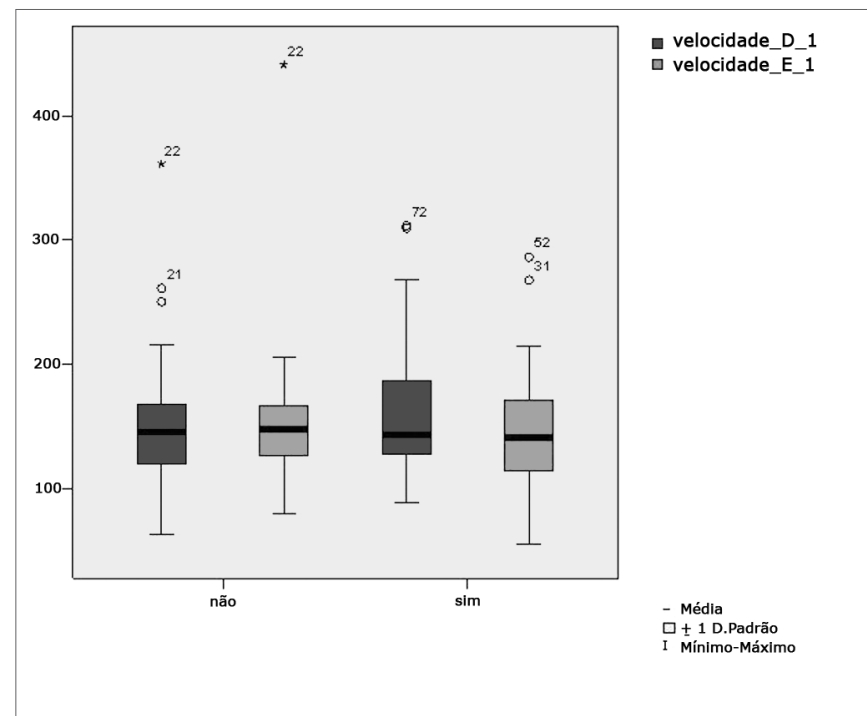

Figura 2. Boxplot para o parâmetro oculomotor de calibração (velocidade) direita e esquerda, por grupo. (Teste t de Student, controlado pelo Teste de Levene para Igualdade de Variâncias, entre as médias das variáveis paramétricas de interesse). 
$\mathrm{Na}$ Figura 3 representamos, em boxplot, o estudo estatístico para o parâmetro oculomotor da calibração: precisão.

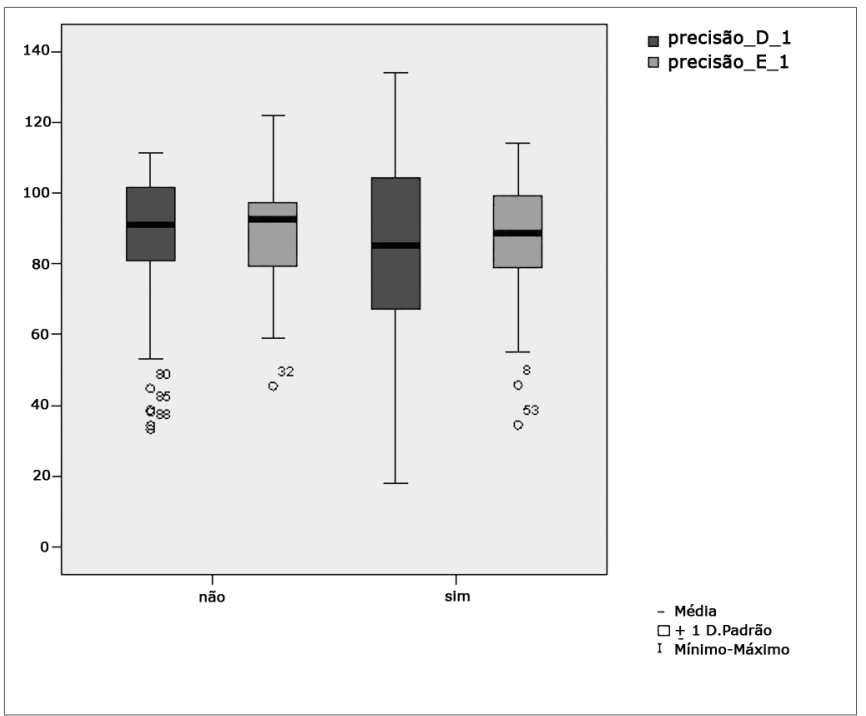

Figura 3. Boxplot para o parâmetro oculomotor de calibração (precisão) direita e esquerda, por grupo. (Teste t de Student, controlado pelo Teste de Levene para Igualdade de Variâncias, entre as médias das variáveis paramétricas de interesse).

Na Tabela 3 pudemos demonstrar o resultado do Teste t de Student, controlado pelo Teste de Levene para Igualdade de Variâncias, com o intuito de verificarmos possíveis diferenças entre as médias das variáveis paramétricas para os parâmetros oculomotores dos movimentos sacádicos: latência; velocidade e precisão.

Tabela 3. Distribuição da amostra em relação aos parâmetros oculomotores dos movimentos sacádicos e desempenho escolar $(n=88)$.

\begin{tabular}{cccccc}
\hline Variável & dificuldade & $\mathrm{n}$ & Média & $\begin{array}{c}\text { Desvio- } \\
\text { padrão }\end{array}$ & $\begin{array}{c}\text { Significân- } \\
\text { cia (p) }\end{array}$ \\
\hline \multirow{2}{*}{ latência_D_2 } & sim & 43 & 215,23 & 98,26 & 0,123 \\
& não & 45 & 176,46 & 58,14 & \\
velocidade_D_2 & sim & 43 & 101,74 & 29,19 & 0,481 \\
& não & 45 & 98,88 & 43,87 & \\
precisão_D_2 & sim & 43 & 95,84 & 25,37 & 0,043 * \\
& não & 45 & 176,77 & 53,51 & \\
latência_E_2 & sim & 43 & 103,26 & 27,67 & 0,274 \\
& não & 45 & 97,91 & 28,58 & \\
velocidade_E_2 & sim & 43 & 108,55 & 33,14 & 0,105 \\
& não & 45 & 176,77 & 53,51 & \\
precisão_E_2 & sim & 43 & 103,26 & 27,67 & 0,274 \\
& não & 45 & 98,40 & 27,41 & \\
\hline
\end{tabular}

A seguir, na Figura 4 apresentamos, em boxplot, o estudo estatístico para o parâmetro oculomotor dos movimentos sacádicos: latência.

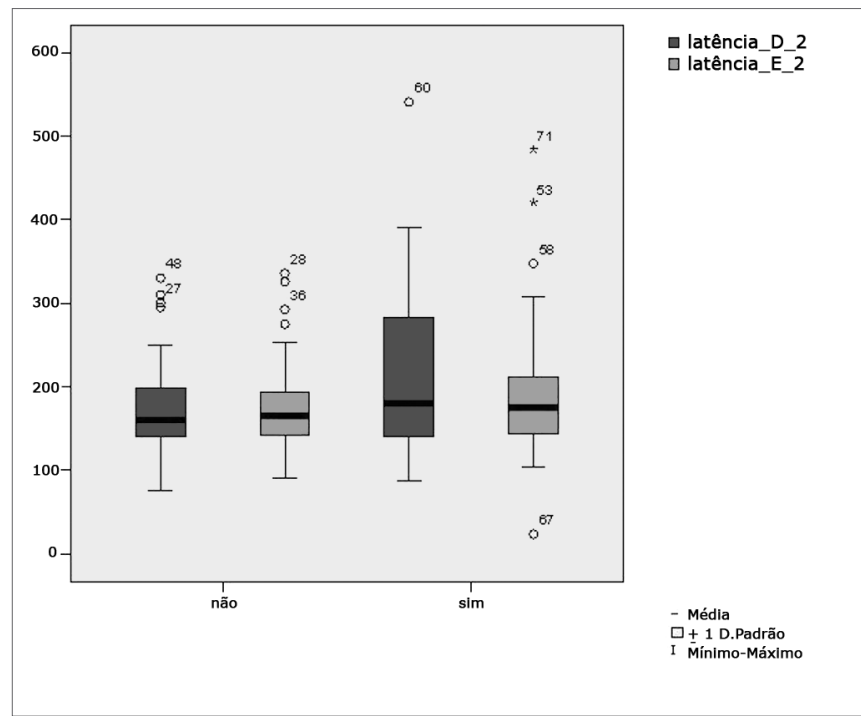

Figura 4. Boxplot para o parâmetro oculomotor dos movimentos sacádicos (latência) direita e esquerda, por grupo. (Teste t de Student, controlado pelo Teste de Levene para Igualdade de Variâncias, entre as médias das variáveis paramétricas de interesse).

Na Figura 5 apresentamos, em boxplot, o estudo estatístico para o parâmetro oculomotor dos movimentos sacádicos: velocidade.

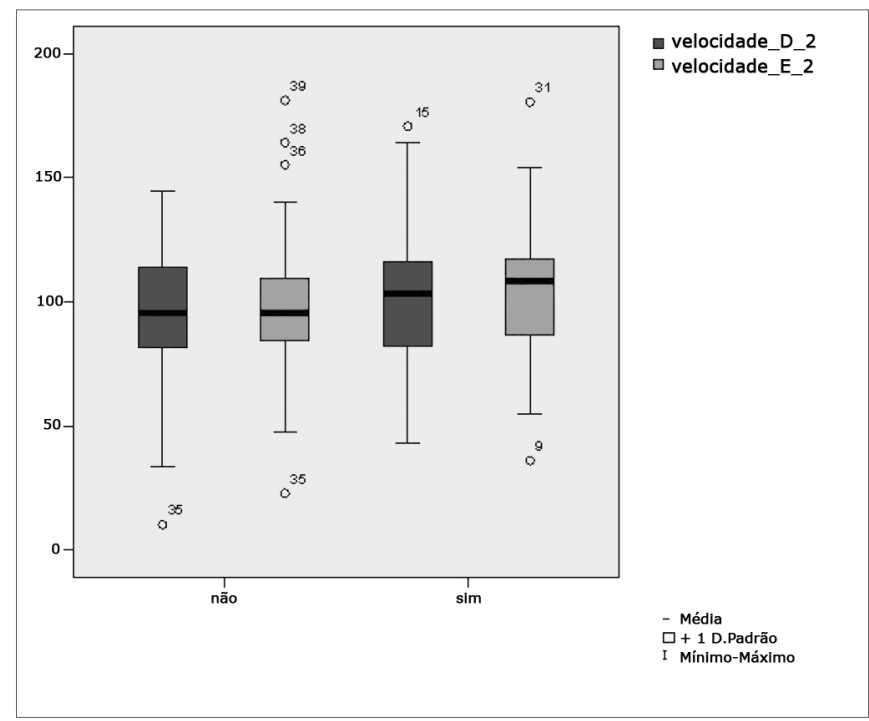

Figura 5. Boxplot para o parâmetro oculomotor dos movimentos sacádicos (velocidade) direita e esquerda, por grupo. (Teste t de Student, controlado pelo Teste de Levene para Igualdade de Variâncias, entre as médias das variáveis paramétricas de interesse).

$a \leq 0,05$ 
Na Figura 6 apresentamos, em boxplot, o estudo estatístico para o parâmetro oculomotor dos movimentos sacádicos: precisão.

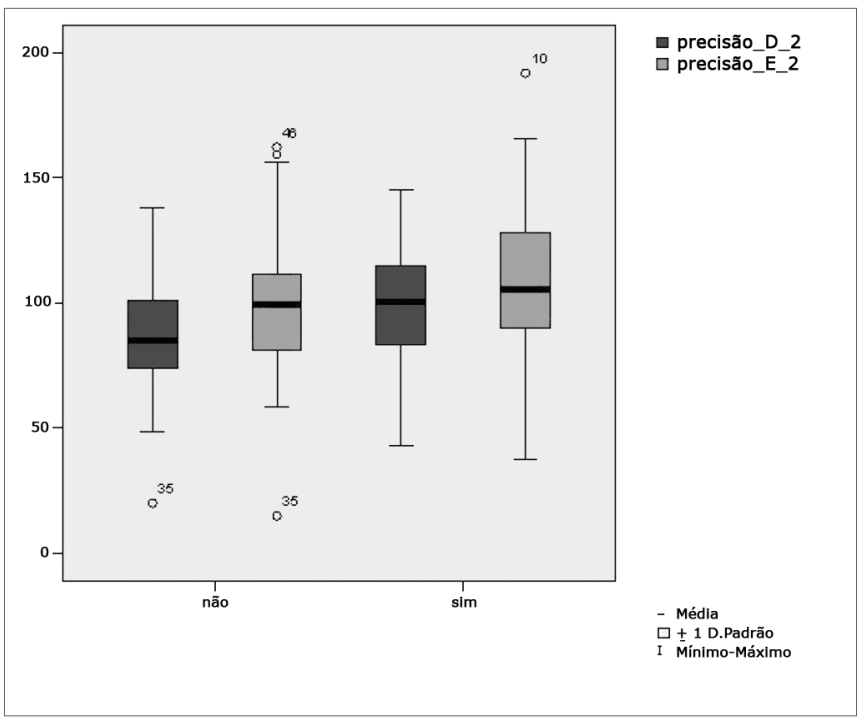

Figura 6. Boxplot para o parâmetro oculomotor dos movimentos sacádicos (precisão) direita e esquerda, por grupo. (Teste t de Student, controlado pelo Teste de Levene para Igualdade de Variâncias, entre as médias das variáveis paramétricas de interesse).

Na Tabela 4 observamos o resultado do Teste t de Student, controlado pelo Teste de Levene para Igualdade de Variâncias, com o intuito de verificarmos possíveis diferenças entre as médias das variáveis paramétricas para os ganhos do rastreio pendular: $20 \mathrm{~Hz} ; 40 \mathrm{~Hz}$ e $80 \mathrm{~Hz}$.

Tabela 4. Distribuição da amostra em relação aos ganhos do rastreio pendular e desempenho escolar $(n=88)$.

\begin{tabular}{cccccc}
\hline Variável & dificuldade & $\mathrm{n}$ & Média & $\begin{array}{c}\text { Desvio- } \\
\text { padrão }\end{array}$ & $\begin{array}{c}\text { Significância } \\
(\mathrm{p})\end{array}$ \\
\hline \multirow{2}{*}{ ganho_20Hz } & sim & 43 & 0,91 & 0,36 & \\
& não & 45 & 0,85 & 0,25 & 0,786 \\
ganho_40Hz & sim & 43 & 1,01 & 0,21 & \\
& não & 45 & 0,98 & 0,22 & 0,695 \\
ganho_80Hz & sim & 43 & 0,86 & 0,23 & \\
& não & 45 & 0,82 & 0,19 & 0,404 \\
\hline
\end{tabular}

$\mathrm{a} \leq 0,05$

A seguir, na Figura 7 apresentamos, em boxplot, o estudo estatístico para os ganhos do rastreio pendular em: $20 \mathrm{~Hz} ; 40 \mathrm{~Hz}$ e $80 \mathrm{~Hz}$.

Na Tabela 5 observamos o resultado do Teste t de Student, controlado pelo Teste de Levene para Igualdade de Variâncias, para verificarmos possíveis diferenças entre as médias das variáveis paramétricas para a preponderância direcional do nistagmo (PDN) na prova optocinética.

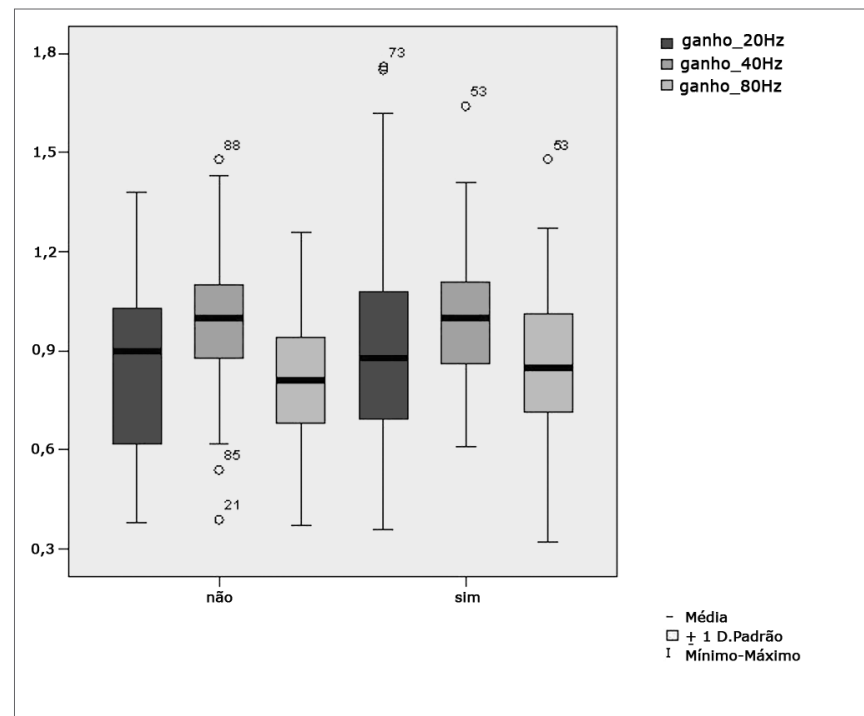

Figura 7. Boxplot para o parâmetro: ganho, do rastreio pendular em: $20 \mathrm{~Hz} ; 40 \mathrm{~Hz}$ e $80 \mathrm{~Hz}$, por grupo. (Teste t de Student, controlado pelo Teste de Levene para Igualdade de Variâncias, entre as médias das variáveis paramétricas de interesse).

Tabela 5. Distribuição da amostra em relação à preponderância direcional do nistagmo (PDN) na prova optocinética e desempenho escolar $(n=88)$.

\begin{tabular}{cccccc}
\hline Variável & dificuldade & $\mathrm{n}$ & Média & $\begin{array}{c}\text { Desvio- } \\
\text { padrão }\end{array}$ & $\begin{array}{c}\text { Significância } \\
(\mathrm{p})\end{array}$ \\
\hline \multirow{2}{*}{ OPTO_PDN } & sim & 43 & 6,20 & 4,55 & 0,486 \\
& não & 45 & 6,05 & 5,29 & \\
\multirow{2}{*}{ VACL_D } & sim & 43 & 11,34 & 2,04 & 0,075 \\
& não & 45 & 10,55 & 2,37 & \\
VACL_E & sim & 43 & 10,92 & 2,01 & 0,486 \\
\hline
\end{tabular}

$a \leq 0,05$

Na Figura 8 apresentamos, em boxplot, o estudo estatístico para a preponderância direcional do nistagmo (PDN) na prova optocinética.

Na Tabela 6 observamos o resultado do Teste t de Student, controlado pelo Teste de Levene para Igualdade de Variâncias. Verificarmos possíveis diferenças entre as médias das variáveis paramétricas para a preponderância direcional do nistagmo (PDN) na prova rotatória pendular decrescente (PRPD), tanto para os canais semicirculares laterais (PDN L), posteriores (PDN P) quanto para os superiores (PDN S).

Na Figura 9 apresentamos, em boxplot, o estudo estatístico para a preponderância direcional do nistagmo (PDN) na prova rotatória pendular decrescente (PRPD), tanto para os canais semicirculares laterais (PDN L), posteriores (PDN P) quanto para os superiores (PDN S).

Na Tabela 7 observamos o resultado do Teste t de 


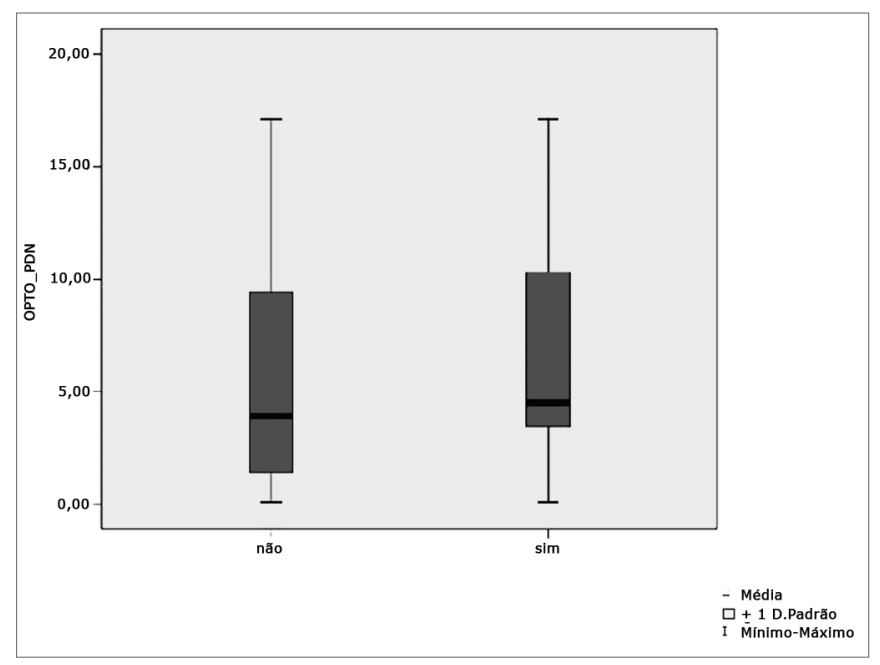

Figura 8. Boxplot para a preponderância direcional do nistagmo (PDN), na prova optocinética por grupo. (Teste t de Student, controlado pelo Teste de Levene para Igualdade de Variâncias, entre as médias das variáveis paramétricas de interesse).

Tabela 6. Distribuição da amostra em relação à preponderância direcional do nistagmo (PDN) na prova rotatória pendular decrescente (PRPD) e desempenho escolar $(n=88)$.

\begin{tabular}{lccccc}
\hline Variável & dificuldade & $\mathrm{n}$ & Média & $\begin{array}{c}\text { Desvio- } \\
\text { padrão }\end{array}$ & $\begin{array}{c}\text { Significância } \\
(\mathrm{p})\end{array}$ \\
\hline \multirow{2}{*}{ PDN_L } & sim & 43 & 10,42 & 7,63 & 0,133 \\
& não & 45 & 12,94 & 7,82 & \\
PDN_P & sim & 43 & 13,70 & 8,32 & 0,726 \\
& não & 45 & 13,01 & 7,55 & \\
PDN_S & sim & 43 & 13,31 & 8,74 & 0,582 \\
& não & 45 & 12,12 & 7,62 & \\
\hline
\end{tabular}

$a \leq 0,05$

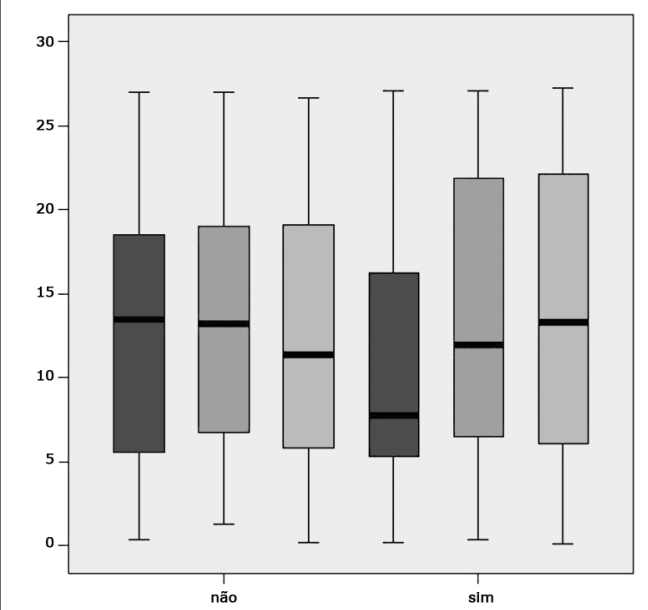

- Média I Mínimo-Máxim

Figura 9. Boxplot para a preponderância direcional do nistagmo (PDN), na prova rotatória pendular decrescente (PRPD) por grupo. (Teste t de Student, controlado pelo Teste de Levene para Igualdade de Variâncias, entre as médias das variáveis paramétricas de interesse).
Tabela 7. Distribuição da amostra em relação VACL na prova calórica, para as temperaturas de $42^{\circ} \mathrm{C}$ e $18^{\circ} \mathrm{C}$ em ambas as orelhas $\mathrm{e}$ desempenho escolar $(n=88)$.

\begin{tabular}{cccccc}
\hline Variável & dificuldade & $\mathrm{n}$ & Média & $\begin{array}{c}\text { Desvio- } \\
\text { padrão }\end{array}$ & Significância $(p)$ \\
\hline \multirow{2}{*}{ PC } & sim & 43 & 23,34 & 13,30 & 0,012 * \\
& não & 45 & 16,76 & 9,65 & \\
D_42 & sim & 43 & 11,00 & 7,04 & 0,478 \\
& não & 45 & 8,81 & 3,04 & \\
E_42 & sim & 43 & 10,99 & 5,44 & 0,425 \\
& não & 45 & 9,67 & 4,01 & \\
D_18 & sim & 43 & 20,02 & 10,29 & 0,012 * \\
& não & 45 & 14,69 & 7,36 & \\
E_18 & sim & 43 & 17,66 & 12,13 & 0,031 * \\
& não & 45 & 12,43 & 6,56 & \\
\hline
\end{tabular}

$a \leq 0,05$

Student, controlado pelo Teste de Levene para Igualdade de Variâncias, com o intuito de verificarmos possíveis diferenças entre as médias das variáveis paramétricas para a velocidade angular da componente lenta do nistagmo (VACL) na prova calórica, tanto para a temperatura $42^{\circ} \mathrm{C}$ quanto para a temperatura de $18^{\circ} \mathrm{C}$ em ambas as orelhas.

A seguir, na Figura 10 apresentamos, em boxplot, o estudo estatístico para a velocidade angular da componente lenta do nistagmo (VACL) na prova calórica, tanto para a temperatura $42^{\circ} \mathrm{C}$ quanto para a temperatura de $18^{\circ} \mathrm{C}$ em ambas as orelhas.

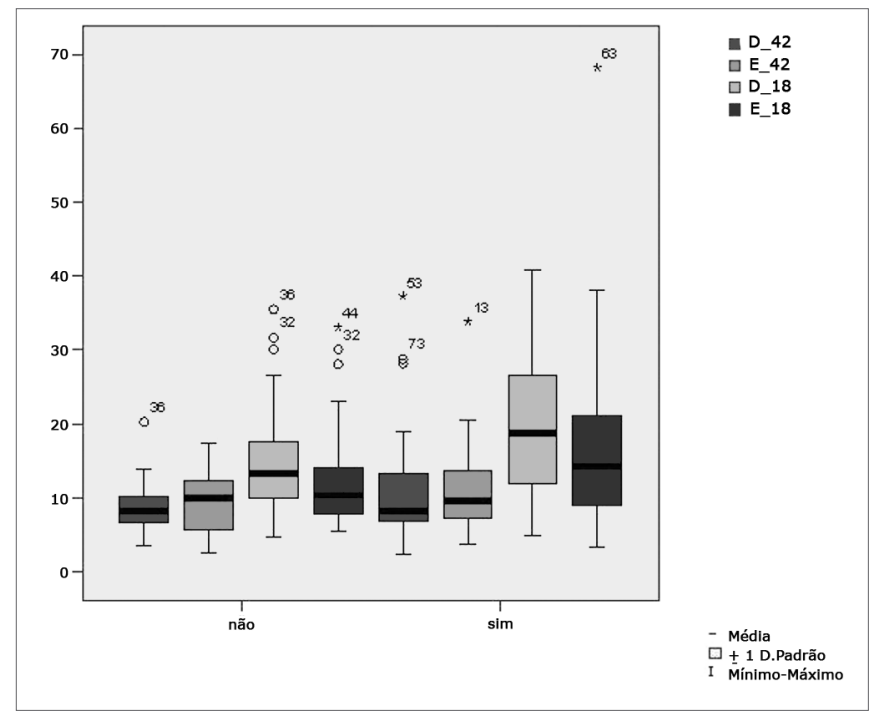

Figura 10. Boxplot para prova calórica (PC) direita e esquerda, em $18^{\circ} \mathrm{C}$ e $42^{\circ} \mathrm{C}$, por grupo. (Teste t de Student, controlado pelo Teste de Levene para Igualdade de Variâncias, entre as médias das variáveis paramétricas de interesse). 
Na Tabela 8 pode ser visualizada a distribuição da amostra quanto aos percentuais do diagnóstico final em função do desempenho escolar. Ao aplicar o Teste de Mann-Whitney, para as diferenças, pudemos notar que tais dados mantêm uma relação estatisticamente significante entre as variáveis estudadas $(\mathrm{p}<0,001)$.

Tabela 8. Distribuição dos percentuais do diagnóstico final em função do desempenho escolar $(n=88)$.

\begin{tabular}{|c|c|c|c|c|c|c|}
\hline \multirow{2}{*}{$\begin{array}{l}\text { Dificuldade } \\
\text { escolar }\end{array}$} & \multicolumn{5}{|c|}{ Diagnóstico final } & \multirow[t]{2}{*}{ Total } \\
\hline & EVN & SVPI & SVPIB & SVPID & SVPIE & \\
\hline \multirow{2}{*}{ Não } & 33 & 2 & 6 & 3 & 1 & 45 \\
\hline & $73,3 \%$ & $4,4 \%$ & $13,3 \%$ & $6,7 \%$ & $2,2 \%$ & $100,0 \%$ \\
\hline \multirow{2}{*}{ Sim } & 14 & 4 & 11 & 8 & 6 & 43 \\
\hline & $32,6 \%$ & $9,3 \%$ & $25,6 \%$ & $18,6 \%$ & $14,0 \%$ & $100,0 \%$ \\
\hline \multirow{2}{*}{ Total } & 47 & 6 & 17 & 11 & 7 & 88 \\
\hline & $53,4 \%$ & $6,8 \%$ & $19,3 \%$ & $12,5 \%$ & $8,0 \%$ & $100,0 \%$ \\
\hline
\end{tabular}

$\mathrm{p}<0,001$ *

Legenda:

EVN = Exame vestibular normal;

SVPI = Síndrome vestibular periférica irritativa;

SVPIB = Síndrome vestibular periférica irritativa bilateral;

SVPID = Síndrome vestibular periférica irritativa à direita;

SVPIE = Síndrome vestibular periférica irritativa à esquerda.

\section{DISCUSSÃO}

Em nossos resultados, foi possível verificar a distribuição da amostra quanto aos percentuais do desempenho escolar em função do sexo. Pudemos notar uma semelhança $(p=0,121)$ entre as variáveis estudadas não se observando, portanto, nenhuma diferença significante entre os dois sexos.

Mediante os dados apresentados notamos que, das 88 crianças avaliadas, 45 (51,0\%) não relataram dificuldades na aprendizagem escolar e 43 (49,0\%) referiram ter dificuldades na aprendizagem escolar.

Observamos dados semelhantes aos estudos de Farias et $\mathrm{al}^{2}{ }^{2}$ que avaliaram 103 crianças com e sem dificuldades escolares verificando que, $58,2 \%$ das crianças não relataram dificuldades escolares e $41,7 \%$ das crianças relataram dificuldades escolares e aos estudos de Narciso et al. ${ }^{7}$ que ao pesquisarem alterações vestibulares em crianças, observaram que $47 \%$ delas apresentavam queixas no desempenho escolar.

Deve-se levar em consideração as argumentações de Schachter et al. ${ }^{3}$ e Sandler ${ }^{4}$ que afirmaram, em seus estudos, que o aprendizado pode ser afetado por fatores genéticos, afecções sensoriais periféricas, afecções neurogênicas, entre outras, e que dentro das perturbações neurogênicas temos os transtornos das integrações funcionais básicas, do esquema corporal e da orientação espacial.
O equilíbrio estático e dinâmico segundo Guardiola et al. ${ }^{5}$, são funções neurológicas importante para a manutenção de posturas adequadas, imprescindiveis no ato de aprender. Em seu estudo, esses autores consideraram que crianças com imaturidade destas funções têm mais probabilidade de apresentar dificuldades de aprendizado.

Grandes contribuições foram dadas pelos trabalhos de Capovilla et al. ${ }^{6}$ e Campos et al. ${ }^{8}$ que consideraram que o sistema vestibular parece estar grandemente envolvido no processo de aprendizagem escolar e que a disfunção vestibular infantil pode afetar consideravelmente a habilidade de comunicação, o estado psicológico e o desempenho escolar, lembrando que o baixo rendimento escolar pode ser um indício valioso de possível labirintopatia.

De acordo com Tuma et al. ${ }^{10}$, a vectonistagmografia digital é um dos métodos mais empregados no nosso meio para avaliar a função vestibular, conferindo maior sensibilidade diagnóstica por permitir a medida dos parâmetros da função vestíbulo-oculomotora à comparação entre estímulos e respostas, além de identificar a direção dos fenômenos.

Em nossos estudos pudemos verificar que as médias encontradas nos parâmetros oculomotores, tanto na calibração dos movimentos oculares quanto nos movimentos sacádicos, estão dentro dos limites de normalidade para a avaliação vectonistagmográfica digital seguindo os valores preconizados por Ganança et al. ${ }^{24}$, no que se refere aos parâmetros latência, velocidade e precisão. Entretanto, verificamos uma relação estatisticamente significante quando observamos as médias, no parâmetro precisão $(p=0,043)$ nos movimentos sacádicos, quando comparadas às crianças com dificuldades escolares e as crianças sem dificuldades escolares. É cauteloso considerar que a significância encontrada ocorreu em um único parâmetro, apenas em um dos lados observados, ou seja, à direita.

Marchesin et al. ${ }^{13}$, em seus estudos, divulgaram que anormalidades no controle voluntário da sacade foram observadas em muitas desordens do desenvolvimento, tais como a dislexia, dificuldades de aprendizagem, hiperatividade e déficit de atenção, afirmando a veracidade dos dados comparados.

Os dados coletados aproximam-se da hipótese aventada por Frank e Levinson ${ }^{15}$ de que uma disfunção vestibular poderia interferir nas fixações oculares seqüenciais necessárias à leitura.

Tais preocupações têm norteados trabalhos como os de Horak et al. ${ }^{16}$ que estudaram a função vestibular em crianças com e sem dificuldades de leitura e escrita, encontrando alteração no reflexo vestíbulo ocular em 20\% das crianças com dificuldades escolares. Esses dados fundamentalizam pesquisas como as de $\mathrm{Hoyt}^{14}$, que refere que o movimento ocular necessário para uma leitura requer movimentos alternados de sácade e períodos de fixação, exigindo uma perfeita integridade do aparelho vestibular e seus movimentos sacádicos. 
Em nossos estudos pudemos verificar que as médias encontradas na prova calórica estão dentro dos limites de normalidade para a avaliação vectonistagmográfica digital, obedecendo aos valores preconizados por Ganança et al. ${ }^{24}$ no que se refere às estimulações térmicas quentes $\left(42^{\circ} \mathrm{C}\right)$, tanto nas crianças com dificuldades escolares quanto nas crianças sem dificuldades. Entretanto, verificamos valores superiores aos limites de normalidade segundo Ganança et al. ${ }^{24}$, nas estimulações térmicas frias $\left(18^{\circ} \mathrm{C}\right)$, quando avaliadas as crianças com dificuldades escolares, apresentando uma relação estatisticamente significante entre as variáveis tanto na estimulação à esquerda quanto à direita $(\mathrm{p}=0,031$ e $\mathrm{p}=0,012$ respectivamente).

A estimulação térmica, realizada por meio da prova calórica, é a prova de maior sensibilidade na bateria de testes vestibulares convencionais. Ela possibilita o encontro de alterações vestibulares em pacientes sem queixas específicas e sem anormalidades nas outras etapas da avaliação vestibular.

A afirmativa exposta acima constituiu pressupostos que conduziram Ganança et al. ${ }^{11}$ a confirmarem, em sua pesquisa, que a prova calórica é o teste vestibular que apresenta o maior número de alterações.

Quando estudamos a preponderância labiríntica ou direcional do nistagmo apresentadas na prova calórica verificamos uma relação estatisticamente significante entre as variáveis ao compararmos as crianças com dificuldades escolares e as crianças sem dificuldades escolares $(\mathrm{p}=$ $0,012)$. Nossos dados revelaram uma disfunção vestibular periférica frente à excitação labiríntica provocando hiperatividade vestibular.

Ganança ${ }^{19}$ realizou um estudo com 64 crianças com distúrbio de linguagem e sem queixas de tonturas encontrando alta incidência de alterações vestibulares de topodiagnóstico periférico.

Com o intuito de determinarmos o diagnóstico final das crianças avaliadas em função do desempenho escolar encontramos alto índice de exame vestibular normal $(73,3 \%)$ nas crianças sem dificuldades escolares e baixo índice de normalidade, nas crianças com dificuldades escolares $(32,6 \%)$. Todas as alterações vestibulares encontradas foram de origem periférica irritativa tanto unilateral quanto bilateral, perfazendo um total de $67,4 \%$ para as crianças com dificuldades escolares e um total de $26,7 \%$ para crianças sem dificuldades escolares. Os dados revelaram uma relação estatisticamente significante entre as variáveis $(\mathrm{p}=0,001)$.

Nossos resultados vão ao encontro aos estudos de Ganança et al. ${ }^{11}$ que estudaram 30 crianças com queixas de dificuldades escolares e encontraram alterações à nistagmografia computadorizada em $20,0 \%$ das crianças avaliadas, sendo todas elas de topodiagnóstico periférico.

Dados semelhantes também foram encontrados nos estudos de Franco e Caetanelli ${ }^{20}$ que realizaram avaliação vestibular em crianças em idade escolar por meio da vectonistagmografia computadorizada e concluíram que 20,7\% delas, apresentaram alterações vestibulares, sendo de caráter periférico irritativo tanto unilateral quanto bilateral.

Os estímulos sensoriais são requisitos fundamentais para o desenvolvimento e aprendizagem da criança. Estimulada desde cedo a criança terá boa coordenação, concentração e será criativa, tendo grandes chances de tornar-se um ser pensante, autoconfiante sem dificuldades na aprendizagem.

Considerando a extrema relevância entre as dificuldades escolares e o sistema vestibular, é clara a necessidade de mais investigações científicas para confirmar os dados coletados neste estudo e, também, para esclarecer os aspectos ambíguos, para os quais respostas não foram encontradas.

Para que aspectos obscuros não impeçam uma adequada intervenção torna-se imprescindível o conhecimento cada vez mais aprofundado valorizando os trabalhos na área da fonoaudiologia.

\section{CONCLUSÃO}

Na presente pesquisa pudemos concluir que asmédias do parâmetro precisão (na avaliação dos movimentos sacádicos) e os limites de normalidade na prova calórica e especialmente na estimulação térmica fria $\left(18^{\circ} \mathrm{C}\right)$, apresentam uma relação estatisticamente significante em crianças com queixas de dificuldades escolares. Os dados revelam uma relação estatisticamente significante de alterações vestibulares em crianças com queixas de dificuldades escolares. Todas as alterações vestibulares encontradas são de origem periférica irritativa.

\section{REFERÊNCIAS BIBLIOGRÁFICAS}

1. Rotta NT, Guardiola A. Distúrbios de aprendizagem. In: Diament A, Cypel S. Neurologia Infantil. 3.Ed. São Paulo: Ateneu, 1996.p. 1062-74

2. Farias LS, Toniolo IF, Coser PL. P300: avaliação eletrofisiológica da audição em crianças sem e com repetência escolar. Rev Bras Otorrinolaringol 2004;70(2):194-9.

3. Schachter DC, Pless IB, Bruck M. Self-report of family histories of learning difficulties. Can J Psychiatry 1992;37:29-32.

4. Sandler AD, Watson TE, Footo M, Levine MD, Coleman WL, Hooper SR. Neurodevelopmental study of writing disorders in middle childhood. J Dev Behav Pediatr 1992;13:17-23.

5. Guardiola A, Ferreira LTC, Rotta NT. Associação entre desempenho das funções corticais e alfabetização em uma amostra de escolares de primeira série de Porto Alegre. Arq Neuropsiquiatr 1998;56(2):281-8.

6. Capovilla AGS, Miyamoto NT, Capovilla FC. Alterações de equilíbrio e nistagmo pós-rotatório em crianças com dificuldades de leitura. Rev Fisioter Univ São Paulo 2003;10(2):61-9.

7. Narciso AR, Zeigelboim BS, Alvarenga KF, Jacob L, Costa Filho AO, Ribas A. Alterações vestibulares em crianças enxaquecosas. Arq Otorrinolaringol 2004;8(3):201-06. 
8. Campos MI, Ganança FF, Caovilla HH, Ganança MM. Prevalência de sinais de disfunção vestibular em crianças com vertigem e/ou outros tipos de tontura. RBM-ORL 1996;3(3):165-70.

9. Caovilla HH, Ganança MM, Munhoz MSL, Silva MLG, Ganança FF, Frazza MM. Vertigem paroxística benigna da infância. In: Silva MLG, Munhoz MSL, Ganança MM, Caovilla HH. Quadros clínicos otoneurológicos mais comuns. São Paulo: Atheneu; 2000.p.109-17.

10. Tuma VC, Ganança CF, Ganança MM, Caovilla HH. Avaliação oculomotora em pacientes com disfunção vestibular periférica. Rev Bras Otorrinolaringol 2006;72(3):407-13.

11. Ganança CF, Pupo AC, Caovilla HH, Ganança MM. Disfunção vestibular em crianças e adolescentes com mau rendimento escolar. Revista Fono Atual 2000;11:21-27.

12. Caovilla HH, Ganança MM, Munhoz MSL, Silva MLG. Equilibriometria Clínica. São Paulo: Atheneu; 1999

13. Marchesin VC, Caovilla HH, Ganança MM. Dos movimentos oculares sacádicos em crianças com desordens do processamento auditivo. Acta ORL 2005;23(2):7-12.

14. Hoyt CS. - Visual training and reading. Am Orthopt J 1999;49:23 23.

15. Frank J, Levinson H. Dysmetric dyslexia and dyspraxia.J Am Acad Child Psychiatry 1973;12: 690-701.

16. Horak FG, Shumway-Cook A, Crowe TK, Black FO. Vestibular function and motor proficiency of children with impaired hearing, or with learning disability and motor impairment. Dev Med Child Neurol 1988;30: 64-79.
17. Ganança MM, Caovilla HH, Munhoz MSL, Silva MLG, Frazza MM As etapas da equilibriometria. In: Caovilla HH, Ganança MM, Munhoz MSL, Silva MLG. Equilibriometria Clínica. São Paulo: Atheneu, 1999.p.68.

18. Ayres AJ. Learning disabilitiesand the vestibular system. J Learn Disabil 1978;11:30-41.

19. Ganança MM. Da vestibulometria em crianças com distúrbio de linguagem. [Tese de Doutorado] São Paulo: Universidade Federal de São Paulo - UNIFESP - EPM; 1989.

20. Franco ES, Caetanelli EB. Avaliação vestibular em crianças sem queixas auditivas e vestibulares, por meio da vectonistagmografia computadorizada. Arq Int Otorrinolaringol 2006;10(1):46-54

21. Glorig A, Davis H. Age, noise and hearing loss. Ann Otol (St. Louis) 1961;70:556-74.

22. Mangabeira Albernaz P, Mangabeira Albernaz PL, Mangabeira Albernaz LG, Mangabeira Albernaz Filho P. Otorrinolaringologia prática. 10ª Edição. São Paulo: Sarvier; 1981

23. Mangabeira Albernaz PL, Ganança MM, Caovilla HH, Ito YI, Novo NF, Juliano I. Aspectos Clínicos e Terapêuticos das Vertigens. Acta WHO 1986;5(Supl 2):49-109.

24. Ganança CF, Souza JAC, Segatin LA, Caovilla HH, Ganança MM. Limites de normalidade dos parâmetros de avaliação a vectonistagmografia digital neurograff. Acta AWHO 2000;2:105.

25. Vieira S. Bioestatística: tópicos avançados. Rio de Janeiro: Elsevier; 2004.

26. Callegari-Jacques SM. Bioestatística: princípios e aplicações. Porto Alegre: Artmed; 2003. 